\title{
Unusual vasomotor coronary arterial responses after reversal of ergonovine induced spasm with glyceryl trinitrate
}

\author{
RYUICHI HATTORI, YOSHIKI TAKATSU, YOSHIKI YUI, HIROSHI NONOGI, \\ CHUICHI KAWAI \\ From the Third Division, Department of Internal Medicine, Faculty of Medicine, Kyoto University, Kyoto, fapan
}

SUMMARY Unusual vasomotor responses in the coronary arteries occurred in two patients after the administration of glyceryl trinitrate to relieve spasm induced by ergonovine maleate. In one patient after treatment with glyceryl trinitrate and despite dilatation of the adjoining segments a new severe reduction in diameter occurred distally to the segments of the right coronary artery where the ergonovine induced spasm developed. In the other patient the left anterior descending artery filled very slowly after the completely occlusive spasm of the right coronary artery had resolved with glyceryl trinitrate though the circumflex did not. These vasomotor changes were associated with chest discomfort and electrocardiographic abnormalities in both cases.

These findings indicate that caution is needed when performing provocative tests even after the ergonovine induced spasm has been reversed with glyceryl trinitrate.

The ergonovine maleate test has been widely used and accepted as a sensitive and specific technique for detecting coronary arterial spasm. ${ }^{1-4}$ The vasospasm induced by ergonovine maleate is usually readily reversible by giving glyceryl trinitrate..$^{1-3}$ On the other, hand some investigators have reported a reversible reduction in coronary artery diameter induced by glyceryl trinitrate. ${ }^{56}$ In addition, clinical experience has proved that in certain cases the use of glyceryl trinitrate is not followed by clinical improvement. ${ }^{78}$ We report two cases of unusual coronary artery vasomotor changes after glyceryl trinitrate had been given to relieve spasm induced by ergonovine maleate.

\section{Case reports}

CASE 1

A 40 year old man presented with a two month history of anterior chest pain at rest in the early morning relieved by sublingual glyceryl trinitrate. On admission examination showed a blood pressure of 108/ $70 \mathrm{~mm} \mathrm{Hg}$ and a pulse rate of $68 / \mathrm{min}$. The resting electrocardiogram was normal, and the exercise stress test was negative. The fasting blood sugar concentra-

Requests for reprints to Professor Chuichi Kawai, Third Division, Department of Internal Medicine, Faculty of Medicine, Kyoto University, Kyoto 606, Japan.

Accepted for publication 18 October 1983 tion was $121 \mathrm{mg} / \mathrm{dl}(6.72 \mathrm{mmol} / \mathrm{l})$, and the oral glucose tolerance test showed a diabetic pattern.

Cardiac catheterisation performed without premedication showed normal resting haemodynamic measurements. Selective coronary arteriography was carried out by the Sones method. The right (Fig la) and left coronary arteriograms were normal,

Ergonovine maleate $0.2 \mathrm{mg}$ given intravenously did not precipitate symptoms or ST segments shifts, but sequential narrowing (Fig. 1b) was evident in the proximal segments of the right coronary artery on the repeat arteriogram. Glyceryl trinitrate $0.3 \mathrm{mg}$ was given sublingually and the left coronary artery visualised in multiple projections. Immediately before the injection of the right coronary artery the patient complained of chest pain, and there was slight elevation of the ST segment in lead II. The right coronary arteriogram performed immediately showed a new $90 \%$ reduction in diameter in the mid-portion (Fig. 1c). The adjoining segments were dilated. During the next three minutes the chest pain abated without specific treatment, and the ST segment elevation resolved.

\section{CASE 2}

A 64 year old man had noticed discomfort in the precordium at rest and during ordinary activity in the early morning for nine months before admission. This symptom became more severe and also occurred at 


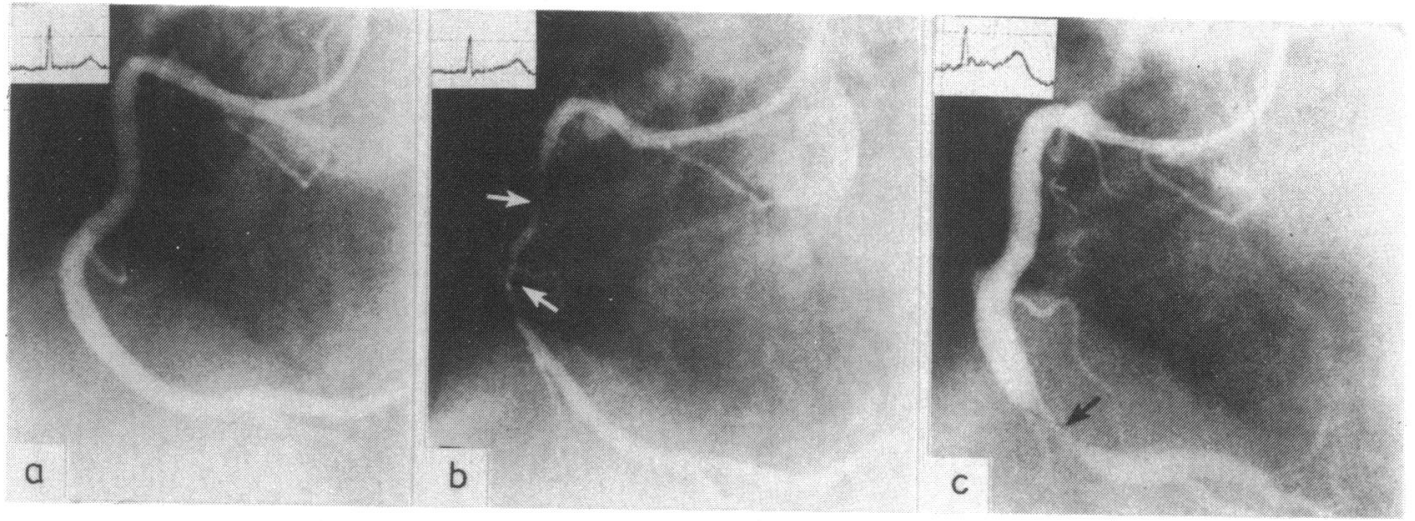

night six months after its onset. He had a long history of systolic hypertension (180-190 mm Hg), which had been successfully treated with hydrochlorothiazide.

On admission his blood pressure was 160/ $64 \mathrm{~mm} \mathrm{Hg}$ and his pulse rate 60 beats/min and regular. A grade 2/6 ejection systolic murmur was present from the apex to the left sternal border. Laboratory examinations produced no useful data.

An electrocardiogram showed left ventricular hypertrophy. Concentric thickening of the left ventricular walls was evident on an echocardiogram. These hypertrophic changes were considered to be the result of previous hypertension. Continuous ambulatory (Holter) electrocardiograms showed ST segment elevation in lead aVF during the episodes of precordial heaviness which were always relieved by sublingual glyceryl trinitrate. Spontaneous episodes were never associated with ST segment elevation in the precordial leads. A treadmill exercise stress test performed in the early morning was positive with ST segment elevation in leads II, III, and aVF, but the same test in the afternoon was negative. Cardiac catheterisation performed without premedication showed a left ventricular pressure of $154 / 14 \mathrm{~mm} \mathrm{Hg}$. The heart rate was 55 beats/min.

Selective coronary arteriography was performed using the Sones technique. The left coronary arteriogram obtained in the right anterior oblique projection was normal. The right coronary artery showed a $40 \%$ narrowing in the proximal segment. The intravenous administration of ergonovine maleate $0.1 \mathrm{mg}$ induced completely occlusive spasm in the proximal segment (Fig. 2a) of the right coronary artery with precordial heaviness and ST segment elevation in leads II, III, and aVF. No delay in filling of the contrast medium was observed in the left coronary arteriogram recorded at the same time. Glyceryl trinitrate $0.3 \mathrm{mg}$ sublingually relieved the discomfort and electrocardiographic changes. After giving glyceryl trinitrate the right coronary artery showed a $40 \%$ narrowing in the proximal segment (Fig. 2b) and the patient again complained of precordial heaviness; the ST segments were elevated in leads V4 and V5. The aortic pressure was $137 / 65 \mathrm{~mm} \mathrm{Hg}$ and the heart rate 60 beats/min. The left coronary arteriogram, which was recorded immediately, showed much slower progression of the contrast medium in the anterior descending artery than in the circumflex artery (Fig. 2c). A further dose of glyceryl trinitrate $0.3 \mathrm{mg}$ was given and gradually the heavy sensation was relieved and the electrocardiographic abnormalities disappeared. No filling delay was noted in the left coronary arteriograms after the chest pain subsided. The left anterior oblique projection alone showed a $25 \%$ narrowing in the proximal portion of the left anterior descending artery (Fig. 2d).

\section{Discussion}

Glyceryl trinitrate is a well known vasodilator, which usually causes generalised coronary arterial dilatation that can be shown by coronary angiography. ${ }^{9}$ Nevertheless, glyceryl trinitrate does not always produce beneficial effects. Dewar and Grimson reported a case of angina pectoris in which glyceryl trinitrate actually induced pain and produced depression of the ST segments at rest comparable to that produced by exercise. ${ }^{7}$ In the study by Russek $e t$ al, using a dose of $0.4 \mathrm{mg}$ of glyceryl trinitrate given sublingually five minutes before exercise, 16 of 158 patients showed even greater electrocardiographic abnormalities than 

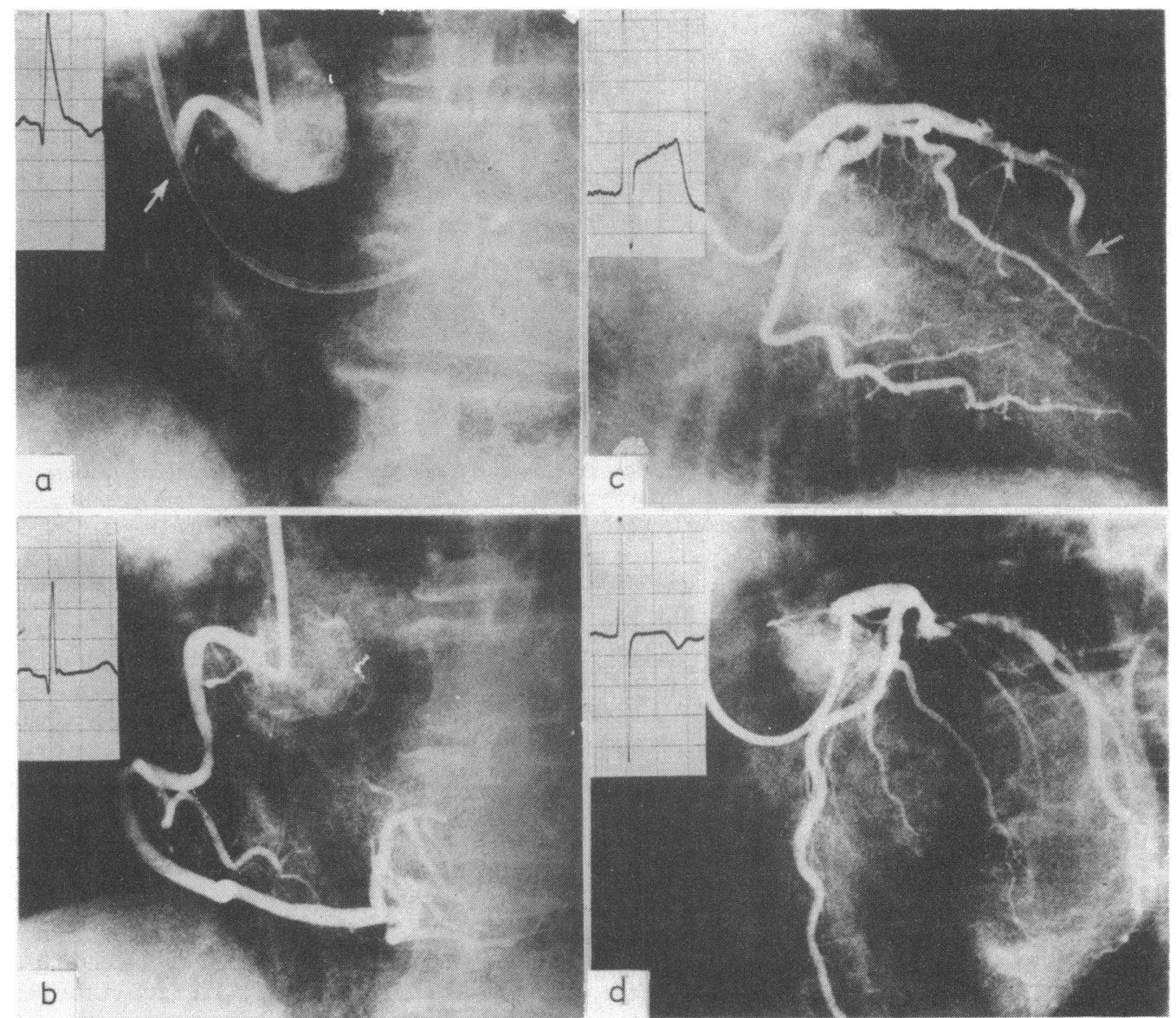

Fig. 2 Case 2: Selected cineangiographic frames of $(a)$ and $(b)$ the right and $(c)$ and $(d)$ the left coronary arteries. Electrocardiographic tracings were lead II in $(a)$ and $(b)$, and lead V4 in $(c)$ and $(d)$. Ergonovine induced completely occlusive spasm ((a) arrow) at the proximal portion of the right coronary artery. This spasm was relieved with glyceryl trinitrate $(b)$. The left coronary arteriogram showed much slower progression of the contrast medium $((c)$ arrow) in the left anterior descending artery than in the lefi circumflex artery. Thisfilling delay disappeared after additional glyceryl trinitrate (d).

those recorded during control exercise tests. ${ }^{8}$ Recently, discrete narrowing of the coronary arteries induced by glyceryl trinitrate has been shown angiographically in three patients. ${ }^{56}$ Of these, one experienced chest pain associated with ST segment elevation, ${ }^{6}$ but the others did not. ${ }^{5}$

In our patients unusual vasomotor responses were observed after glyceryl trinitrate had been given to relieve the coronary artery spasm induced by ergonovine maleate. Such responses accompanied by chest discomfort and electrocardiographic abnormalities after ergonovine maleate tests have not been reported previously.

Spasm induced by ergonovine maleate is usually relieved by glyceryl trinitrate ${ }^{1-3}$ although it may occasionally be refractory. ${ }^{10}$ Indeed segmental narrowing and total obstruction were relieved by glyceryl trinitrate in cases 1 and 2 respectively in our study. In case 1, however, the right coronary arteriogram obtained after sublingual glyceryl trinitrate showed a new severe reduction in diameter in a different segment from that of the ergonovine maleate induced spasm. In case 2, despite the absence of a pronounced fall in blood pressure, the left anterior descending artery filled very slowly simultaneously with a precordial heavy sensation and ST segments elevation in leads V4 and V5. Since no severe stenoses were present in the proximal segment of the left anterior descending artery, delayed filling seemed to be produced by selectively increased vascular resistance, 
possibly induced by diffuse arteriolar constriction.

Glyceryl trinitrate given during spontaneous episodes of angina was not followed by any adverse effects in either patient in this study. These coronary vasomotor changes, therefore, seemed to be induced by the additive effects of ergonovine maleate and glyceryl trinitrate rather than by glyceryl trinitrate alone. On the other hand, these changes may possibly be due simply to the continued action of ergonovine maleate alone. In either case, it is difficult to explain these vasomotor changes. These findings suggest that caution is needed when performing provocative tests even after the spasm induced by ergonovine maleate has been reversed with glyceryl trinitrate.

We thank Dr A S Cary for her critical reading of the manuscript.

\section{References}

1 Schroeder JS, Bolen JL, Quint RA, et al. Provocation of coronary spasm with ergonovine maleate. New test with results in 157 patients undergoing coronary arteriography. Am $\mathcal{F}$ Cardiol 1977; 40: 487-91.

2 Heupler FA Jr, Proudfit WL, Razavi M, Shirey EK,
Greenstreet R, Sheldon WC. Ergonovine maleate provocative test for coronary arterial spasm. Am $\mathcal{f}$ Cardiol 1978; 41: 631-40.

3 Curry RC Jr, Pepine CJ, Sabom MB, Feldman RL, Christie LG, Conti CR. Effects of ergonovine in patients with and without coronary artery disease. Circulation 1977; 56: 803-9.

4 Maseri A, Severi S, De Nes M, et al. "Variant" angina: one aspect of a continuous spectrum of vasospastic myocardial ischemia. Am $\mathcal{F}$ Cardiol 1978; 42: 1019-35.

5 Feldman RL, Pepine CJ, Conti CR. Unusual vasomotor coronary arterial responses after nitroglycerin. Am $\mathcal{F}$ Cardiol 1978; 42: 517-9.

6 Bove AA, Vlietstra RE. Paradoxical angina after nitroglycerin-another variant [Letter]. $N$ Engl f $\mathrm{Med}$ 1982; 306: 484-5.

7 Dewar HA, Grimson TA. Khellin in the treatment of angina of effort. Br Heart $\mathcal{F} 1950$; 12: 54-60.

8 Russek HI, Urbach KF, Island S. Paradoxical action of glyceryl trinitrate (nitroglycerin) in coronary patients. fAMA 1955; 158: 1017-21.

9 Gensini GG. Vasodilators, spasm, and coronary arteriography. In: Coronary arteriography. Mount Kisco, NY: Futura, 1975: 417-35.

10 Buxton A, Goldberg S, Hirshfeld JW, et al. Refractory ergonovine-induced coronary vasospasm: importance of intracoronary nitroglycerin. Am $\mathcal{f}$ Cardiol 1980; 46: $329-34$. 